\title{
Editorial
}

\section{Ambient Intelligence in the Internet of Things}

\author{
Sergio Ricciardi, ${ }^{1}$ Jose Roberto Amazonas, ${ }^{2}$ \\ Francesco Palmieri, ${ }^{3}$ and Maria Bermudez-Edo ${ }^{4}$ \\ ${ }^{1}$ Universitat Politècnica de Catalunya. BarcelonaTech (UPC), Barcelona, Spain \\ ${ }^{2}$ Escola Politécnica da Universidade de S. Paulo, Sao Paulo, SP, Brazil \\ ${ }^{3}$ University of Salerno, Salerno, Italy \\ ${ }^{4}$ Universidad de Granada, Granada, Spain \\ Correspondence should be addressed to Sergio Ricciardi; sergio.ricciardi@ac.upc.edu
}

Received 29 May 2017; Accepted 29 May 2017; Published 22 June 2017

Copyright (C) 2017 Sergio Ricciardi et al. This is an open access article distributed under the Creative Commons Attribution License, which permits unrestricted use, distribution, and reproduction in any medium, provided the original work is properly cited.

Since the beginning of the Information Age, the Information and Communications Technologies (ICT) have been characterized by cyclic transitions between centralized and distributed computing models. From the early mainframes to the personal computers, from the Cloud to the Smartphones, the focus has moved according to the technological advancements and the key applications that have enabled continuous paradigm shifts from centralized computing to thin clients and vice versa.

The Internet of Things (IoT) is not an exception and perpetrates such a trend. The appearance of cheap ubiquitous sensors that can be easily integrated virtually anywhere has accelerated the growth of Ambient Intelligence, which refers to the capacity of the system to sense the environment and to respond to the presence of people and to certain events or conditions, according to the typical situation awareness principia and behaviors. As a result, the IoT has gained a lot of popularity, propelled by the new advancements in mobile information systems. Tiny smart objects are being embedded in everyday things, equipped with enough computational capabilities, and easily interconnected among them and to the Internet. Many cross-domain applications are able to access multiple sensors, actuators, and user-generated data by also controlling them and interoperating among different contexts. Smart things cooperate to provide valuable information and increase the potentialities of their actions and the comfort of people using them. Such improvements have enabled the creation of new applications and scenarios such as Smart Cities and Smart Buildings. Ambient Intelligence will possibly represent one of the biggest advancements in the near future in people's lifestyle, influencing more and more aspects of the society. The way people perceive technology is going to change: as these devices become smaller, more connected, and more integrated into the environment, the traditional ICT technologies become more and more invisible to the common users until only the user interface remains perceivable by them. Users will interact directly or indirectly with the interfaces of the systems that respond to people presence, actions, and decisions, offering integrated solutions and improving the Quality of Experience (QoE). To this end, Mobile Information Systems are crucial for the Ambient Intelligence, since the majority of the sensors and actuators rely on mobile and wireless technologies, distributed information systems, and big data analytics applications to collect, store, and process the data. Such a scenario has been made possible by harmonizing the advancements at different layers: Network (e.g., WSAN, RFID, NFC, BLE, ZigBee, IEEE 802.1lah, and 6LoWPAN), Operating System (e.g., TinyOS, Contiki, and FreeRTOS), Service Layer and Middleware platforms (e.g., FIWARE and CityPulse), among others. However, despite several recent efforts, the IoT development has followed the "vertical silos" paradigm, in which proprietary solutions can hardly interoperate, thus limiting the potentialities offered by such a paradigm. Research and Innovation is needed to address new model-centric and predictive engineering methods and tools 
for cyber-physical systems and "systems of systems" with a high degree of autonomy ensuring adaptability, scalability, complexity management, security and safety, and providing trust to humans in the loop.

The articles contained in the present issue contribute with original research experiences illustrating and stimulating the continuous efforts to understand the implications of the Ambient Intelligence in the IoT. They have been carefully selected according to their subject in order to feature the latest advances and directions in this amazing scenario by exploring the potential of new more intelligent and situationaware architectures, protocols, services, and applications in the next generation IoT environments. We can group the presented experiences in the aforementioned network, middleware, and application layers.

At the network layer, in the paper "The Social Relationship Based Adaptive Multi-Spray-and-Wait Routing Algorithm for Disruption Tolerant Network," J. Guan et al. propose a new routing model in disruption tolerant networks (DTN) that takes into account buffer management and social relationships among nodes. The use of the social properties of nodes is the solution of choice for a novel message routing that is based on message ferrying between separated parts of the network. Basically the work analyzes the spraybased schemes and the applications of social characters in DTN routing and proposes a new algorithm with timeout detection based on ACK messages, with a buffer management mechanism to reduce the node overload, considering the social relationship to select the next node. The proposed algorithm is evaluated in comparison with others well-known algorithms as Epidemic, Spray and Wait, and PropHet. The results are very promising in Shortest Path Map Based mobility model and in SLAW (Self-similar Least Action Walk) mobility model.

In the paper "A Fine-Grained Visible Light Communication Position Detection System Embedded in One-Colored Light Using DMD Projector," M. Kodama and S. Haruyama deal with a recurrent problem in the ubiquity of the IoT: the position detection. Authors design and implement a prototype based on a fine-grained visible light communication (VLC) embedded in one-colored light using Digital Micromirror Device (DMD) projector. Results demonstrating the effectiveness of their method are promising, showing an accuracy of detection in the order of millimeters.

The contribution "Power Allocation Scheme for Femtoto-Macro Downlink Interference Reduction for Smart Devices in Ambient Intelligence" by X. Su et al. presents a power allocation algorithm in the Home evolved NodeB "FemtoCell" LTE environment that considers the Closed Subscriber Group mode, introduced by 3GPP, and a cochannel spectrum policy. By using cross-tier signalto-leakage-plus-noise it reduces the greater part of the cross-tier DL interference from evolved NodeB and provides a reduction of the remaining interference.

At the middleware layer, in the paper "Design and Implementation of a Cloud-Based Platform for Unleashing the Personal and Communal Internet of Things," I. Elicegui et al. propose a social IoT framework for sharing devices and services among people in a community. In their proposal, authors include humans in the loop extending the existing architecture models to accommodate the requirements stemming from the vision of people-sourced IoT devices, which are shared to create applications and services in smart communities. The owners of the shared devices, however, are always empowered to control the access to the shared information. The software platform is based on available generic enablers as defined by the FIWARE initiative.

In the paper "A Collaborative Semantic Annotation System in Health: Towards a SOA Design for Knowledge Sharing in Ambient Intelligence" by G. Guerrero-Contreras et al., the authors state that the conversion of the widespread Content Management Systems (CMS) into its semantic equivalent is a relevant step, as this enables the benefits of the semantic web to be extended. The FLERSA annotation tool converts the Joomla! CMS into its semantic equivalent. However, this tool is highly coupled with that specific Joomla! platform. Ambient intelligent (AmI) environments can be seen as a natural way to address complex interactions between users and their environment and should be transparently supported through distributed information systems. To build distributed information systems for AmI environments it is necessary to make important design decisions and apply techniques at system/software architecture level. In this paper, a SOA-based design solution consisting of two services and an underlying middleware is combined with the FLERSA tool. It allows end-users to collaborate independently of technical details and specific context conditions and in a distributed, decentralized way.

At the application layer, the paper "Active RFID Attached Object Clustering Method with New Evaluation Criterion for Finding Lost Objects" by M. Tanbo et al., the authors propose an active RFID attached object clustering method based on RSSI series for finding lost objects. This approach to find lost objects does not execute existing localization methods. It is hypothesized that users can deduce the location of a lost object from information about surrounding objects in an environment where RFID tags are attached to all personal belongings. To help find lost objects from the proximity between RFID tags, the system calculates the proximity between a pair of RFID tags from the RSSI series and estimates the groups of objects in the neighbourhood. A new method for calculating the proximity of the lost object to those around it is proposed which uses a distance function between RSSI series and estimates the group by hierarchical clustering. The experimental results indicate that the method provides a clear advantage in finding lost objects at low financial and installation cost and can estimate groups accurately even if the smartphone or RSSI sensor is moving quickly.

The work "DrivingSense: Dangerous Driving Behavior Identification Based on Smartphone Autocalibration" by C. $\mathrm{Ma}$ et al. presents DrivingSense, a reliable dangerous driving behavior identification scheme based on a smartphone autocalibration algorithm based on the determination of sensor noise distribution when a vehicle is being driven. The proposed scheme leverages the corrected sensor parameters 
in order to identify three kinds of dangerous behaviors: speeding, irregular driving direction change, and abnormal speed control, resulting in a significant degree of precision and reliability.

Sergio Ricciardi

Jose Roberto Amazonas

Francesco Palmieri

Maria Bermudez-Edo 

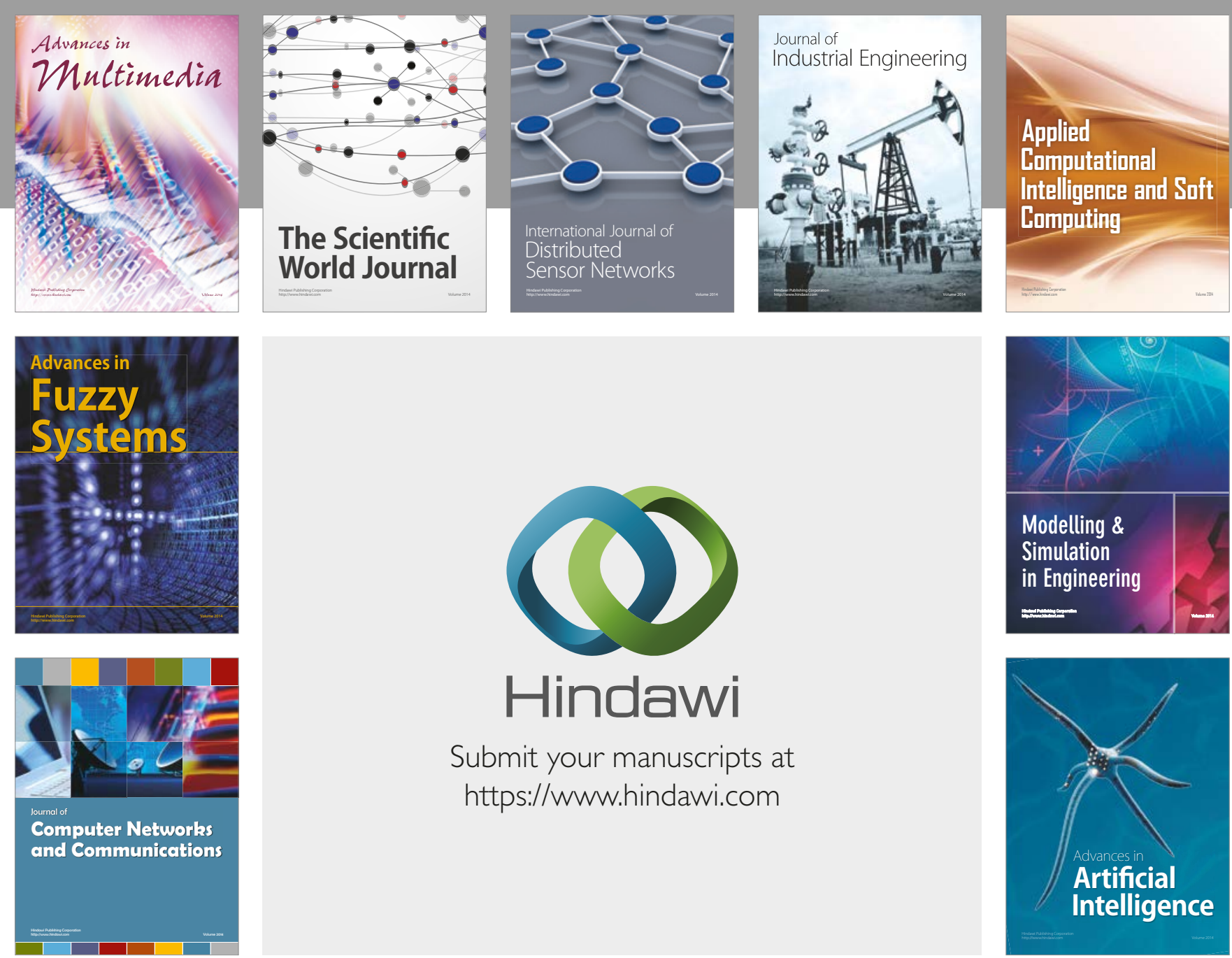

\section{Hindawi}

Submit your manuscripts at

https://www.hindawi.com
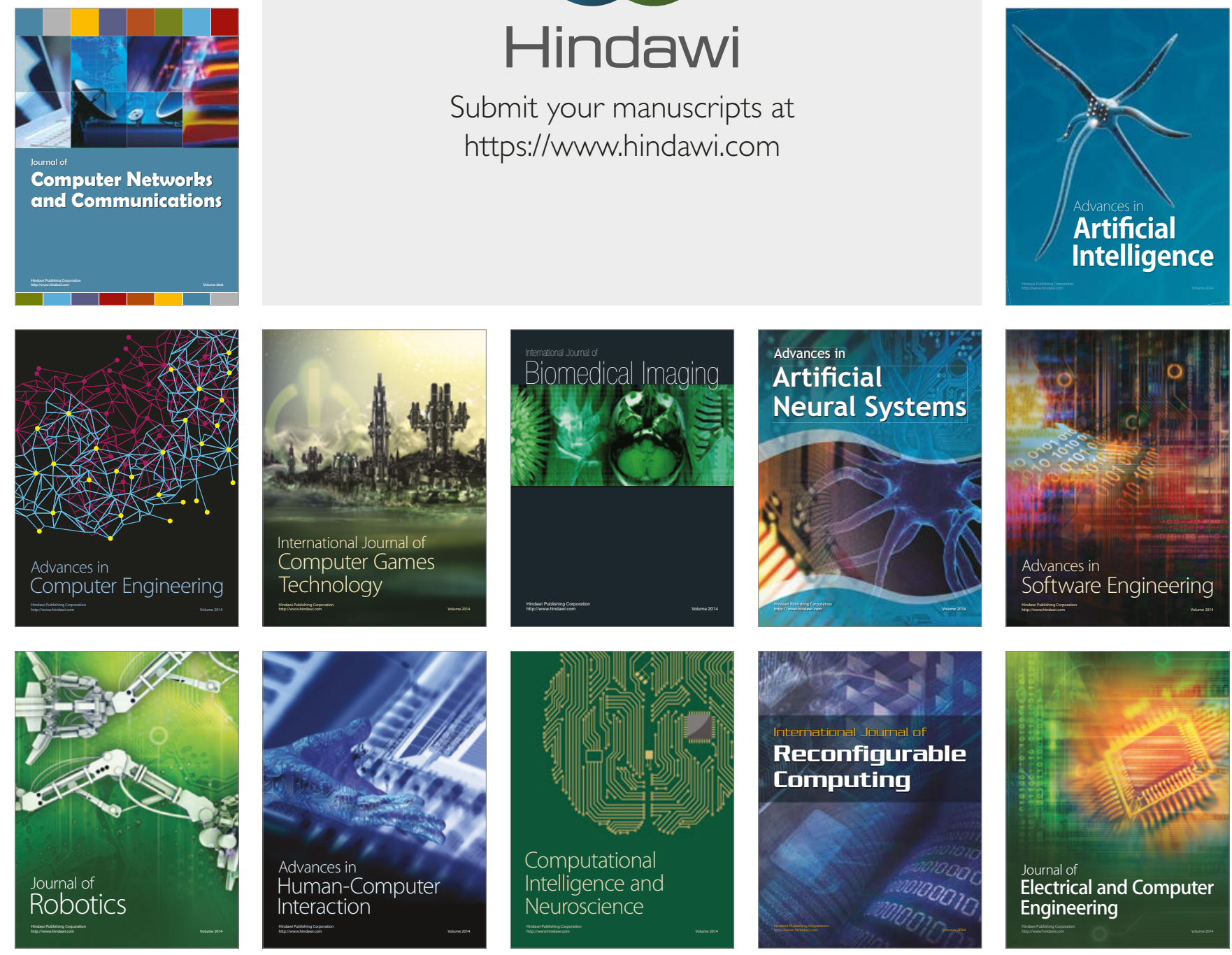